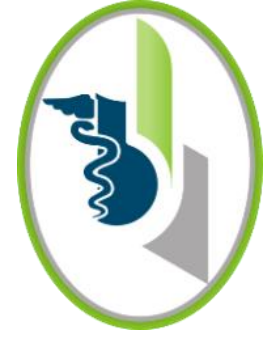

ACCESO ABIERTO

Para citaciones: Gallo, R., Ariza, A. (2021). Indicaciones para la realización de cerclaje profiláctico en la prevención de parto pretérmino del embarazo de feto único. Revista Ciencias Biomédicas, 10(2), 129-135. https://doi.org/10.32997/rcb2021-3373

Recibido: 25 de enero de 2021 Aprobado: 21 de marzo de 2021

Autor de correspondencia:

Anggie Tatiana Ariza

anggie-ariza@hotmail.com

Editor: Inés Benedetti. Universidad de Cartagena-Colombia.

Copyright: (C) 2021. Gallo, R., Ariza, A. Este es un artículo de acceso abierto, distribuido bajo los términos de la licencia https://creativecommons.org/licenses/by-nc$\mathrm{sa} / 4.0 /$ la cual permite el uso sin restricciones, distribución y reproducción en cualquier medio, siempre y cuando el original, el autor y la fuente sean acreditados.

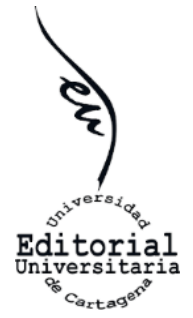

\section{Indicaciones para la realización de cerclaje profiláctico en la prevención de parto pretérmino del embarazo de feto único}

\author{
Indications to perform a prophylactic cerclage to prevent \\ preterm delivery of single fetus pregnancy
}

Roberto Gallo Roa ${ }^{1}$, Anggie Tatiana Ariza Alvis ${ }^{2}$ iD

${ }^{1}$ Departamento de Ginecología y Obstetricia, Hospital Universitario de la Samaritana, Bogotá, Colombia.
Departamento de Ginecología y Obstetricia, Hospital Militar Central, Bogotá, Colombia.
${ }^{2}$ Pregrado, Facultad de Medicina, Universidad Militar Nueva Granada, Bogotá, Colombia.

\section{RESUMEN}

Introducción: el parto pretérmino es una problemática a nivel mundial, la cual lleva a morbimortalidad de los neonatos a corto y a largo plazo. Se han descrito diferentes medidas que favorecen mayor tiempo fetal in útero, favoreciendo un mejor pronóstico neonatal como lo es la sutura cervical denominada cerclaje.

Objetivo: consolidar información sobre las indicaciones de la realización del cerclaje profiláctico en la prevención de parto pretérmino del embarazo de feto único.

Métodos: se realizó una revisión narrativa de la literatura con búsqueda sistemática en bases de datos como Pubmed, Embase y Science Direct. Con términos DeCS: Cerclaje cervical y trabajo de parto prematuro. Aplicando criterios de inclusión y exclusión, así como lectura crítica con herramienta CASPe, fueron seleccionados veintisiete artículos.

Resultados: trece de los artículos revisados artículos consideran el antecedente obstétrico sugestivo de incompetencia cervical como requisito para considerar la prevención con cerclaje, de estos, siete lo consideran indicación única del cerclaje cervical profiláctico y seis consideran este antecedente asociado a disminución de la longitud cervical como indicación del mismo. Los catorce artículos.

Conclusiones: en toda paciente con antecedente obstétrico de riesgo se debe valorar la longitud cervical por ecografía cada semana hasta llegar a término, en caso de longitud menor a $25 \mathrm{~mm}$ o descenso progresivo de la longitud, se debe realizar cerclaje cervical profiláctico. Se pueden considerar medidas farmacológicas como medida de prevención y en caso de antecedentes de alto riesgo se puede considerar indicación única para cerclaje cervical.

Palabras Clave: cerclaje cervical; trabajo de parto prematuro.

\section{ABSTRACT}

Introduction: preterm birth increase neonatal mortality and morbidity in a short and long term. Different measures have been described that favor a longer fetal time in utero, favoring a better neonatal prognosis such as the cervical suture called cerclage. 
Objective: to consolidate information on the indications for performing prophylactic cerclage in the prevention of preterm delivery of a single fetus.

Methods: a narrative review of the literature was carried out with a systematic search in databases such as Pubmed, Embase and ScienceDirect. With DeCS terms "Cerclage, Cervical and premature birth". Applying inclusion and exclusion criteria, as well as critical reading with the CASPe tool, twenty seven articles were selected.

Results: thirteen of the reviewed articles consider the obstetric antecedent suggestive of cervical incompetence as a requirement to consider prevention with cerclage, seven of these consider it sufficient as the only indication and six articles consider this antecedent associated with decreased cervical length as an indication for prophylactic cervical cerclage. The remaining fourteen articles highlight the importance of follow-up with complementary methods.

Conclusions: in all patients with an obstetric history of risk, the cervical length should be evaluated by ultrasound every week until reaching term and in case of cervical length less than $25 \mathrm{~mm}$ or progressive decrease in length, perform prophylactic cervical cerclage. Pharmacological measures can be considered as a preventive measure, in case of a high-risk history it can be considered as indication for cervical cerclage.

Keywords: cervical cerclage; premature birth.

\section{INTRODUCCIÓN}

El parto pretérmino definido como la finalización de la gestación mayor de 20 semanas y menor de 37 semanas, es una problemática presente a nivel mundial, encontrando que anualmente hay aproximadamente 13 millones de nacidos antes del término, condición que favorece la mortalidad y la morbilidad de los mismos tanto a corto como a largo plazo $(1,2)$. Es importante considerar que a mayor tiempo faltante para el término, mayor será la inmadurez neonatal tanto a nivel funcional como orgánica y con ello, un pronóstico desfavorable, puesto que padecer una condición que impide la culminación completa del embarazo se traduce en pausar un desarrollo fisiológico y predeterminado, sometiendo al recién nacido a una transición para la cual no se encuentra preparado (3); teniendo presente que la ganancia de una o dos semanas in útero se puede traducir en la probabilidad de que el neonato sobreviva o no. En Colombia el DANE (Departamento Administrativo Nacional de Estadísticas) reportó en 2017 19.8\% de nacimientos pretérmino, con $0,4 \%$ correspondiente a 2499 nacimientos pretérmino extremo entre las semanas 22 y 27 de gestación, así mismo, el Instituto Nacional de Salud Colombiano reporta la relación del trabajo de parto pretérmino con la mortalidad neonatal presente en el año 2018, donde demuestra ser la principal causa de mortalidad con un $23,5 \%(4,5)$.

Se han descrito diferentes medidas que favorecen mayor tiempo fetal in útero, la cuales pueden no lograr llevar a término la gestación, pero permiten retrasar la finalización de la misma, favoreciendo un mejor pronóstico neonatal (6). Dentro de las diversas posibilidades, se encuentra desde la posibilidad de manejo farmacológico, hasta la realización de procedimientos quirúrgicos como la sutura cervical denominada cerclaje, este es un procedimiento que se empleó en los años cincuenta con la indicación principal en pacientes con insuficiencia cervical, desde entonces se ha adoptado la posibilidad de realizar el procedimiento de forma preventiva o de realizarlo de forma emergente $(7,8)$.

La realización del cerclaje profiláctico en la actualidad es un tema debatido, se deben tener presentes los diferentes criterios para seleccionar adecuadamente los grupos de pacientes que realmente se verán beneficiadas con la realización del procedimiento, esto con la intención de no 
realizar un cerclaje que no beneficie a la paciente y que sume los riesgos que conlleva la realización del procedimiento como, ruptura prematura de membranas, corioamnionitis, hemorragia y laceración cervical, entre otros $(9,10)$. El objetivo del presente estudio fue realizar una revisión en la literatura sobre las indicaciones de la realización del cerclaje profiláctico en la prevención de parto pretérmino del embarazo de feto único.

\section{MÉTODOS}

\section{Estrategia de búsqueda}

Se realizó una revisión narrativa de la literatura con búsqueda sistemática, utilizando recursos de búsqueda avanzada de bases de datos seleccionadas por incluir estudios provenientes del área de las ciencias de la salud y por ser bases de datos reconocidas en el área, tales como Embase, Pubmed, Science Direct, MEDLINE a través de Ovid, Centro de Registros Cochrane de Ensayos Controlados (CENTRAL) y LILACS (Literatura Latinoamericana y del Caribe en Ciencias de la Salud). Los términos MeSH (Medical subject headings) utilizados fueron "Cerclage, Cervical and premature birth", los términos DeCS (Descriptores en Ciencias de la Salud) utilizados, fueron "Cerclaje cervical y trabajo de parto prematuro". Se complementó la información recolectada por medio de la búsqueda de estudios adicionales en las listas de referencias de los artículos seleccionados.

\section{Criterios de inclusión y exclusión}

El criterio de inclusión utilizado consistió en incorporar revisiones sistemáticas, artículos originales, metaanálisis, estudios de casos, incluyendo casos únicos, resúmenes de congresos y artículos de opinión. Se excluyeron los artículos enfocados en gestantes con embarazo múltiple, artículos sobre uso terapéutico y no profiláctico del cerclaje y artículos sin indicación clara para la realización del cerclaje profiláctico, no se estableció un parámetro de fechas de publicación.

\section{Recolección de información}

Fueron encontrados 160 registros posterior a la combinación de las diferentes palabras clave, a los que se les aplicaron los criterios de inclusión, obteniendo 70 artículos, de los que se verificó su disponibilidad en castellano, inglés y francés, posteriormente se realizó una lectura general de los mismos, aplicándoles criterios de exclusión, con un resultado de 45 artículos, sometidos a una fase de lectura crítica, utilizando la herramienta CASPe para aceptar artículos con la suficiente calidad científica, por lo que finalmente fueron seleccionados veintisiete artículos que cumplían con las características establecidas (Figura 1).

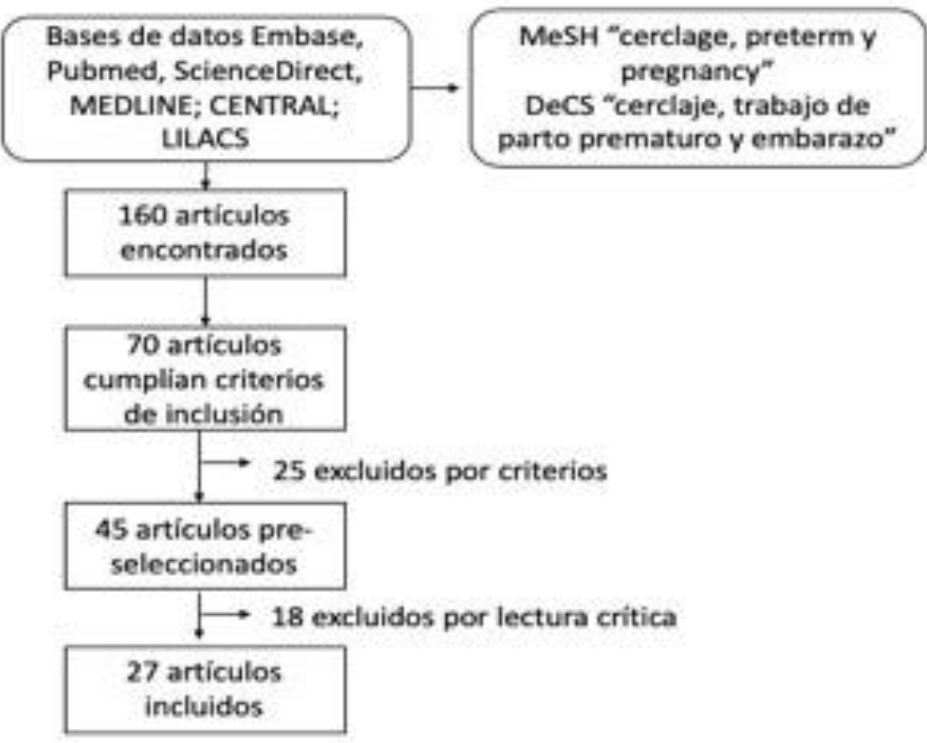

Figura 1. Síntesis del proceso de selección de artículos y recolección de la información. 


\section{RESULTADOS}

Existen diferentes posiciones respecto a las indicaciones de la realización del cerclaje profiláctico en la prevención de parto pretérmino del embarazo de feto único. Todos los autores concluyen que se debe plantear una medida para prevenir el desenlace de la incompetencia cervical teniendo presente el incremento de la morbimortalidad del recién nacido que es proporcional al tiempo restado in útero, asimismo se deben identificar pacientes candidatas para realizar un seguimiento con cervicometría sin someter de forma precipitada a las pacientes a procedimientos no justificados. En la Tabla 1 se exponen los principales estudios analizados, dentro de los cuales, trece artículos respaldaron la idea del requerimiento de un antecedente obstétrico sugestivo de incompetencia cervical como indicación para tratar con cerclaje cervical la incompetencia cervical y así prevenir el parto pretérmino; de estos artículos se encontraron siete $(5,12,13,14,15,19,20)$ que consideran este antecedente suficiente como indicación única, y seis artículos $(3,17,18,21,23,24)$ que consideran que este antecedente deber estar asociado a disminución de la longitud cervical para ser considerado como indicación de cerclaje cervical profiláctico. Por otra parte, los catorce artículos restantes $(1,2,4,6,7,8,9,10,11,16,22,25,26,27)$, resaltan la importancia de no exponer a un riesgo precipitado a la gestante y no someterla a un procedimiento quirúrgico con probabilidades de complicaciones derivados del mismo, sin un sustento soportado por valoraciones seriadas de la longitud cervical con disminución progresiva de la misma demostrada.

Tabla 1. Indicaciones para el cerclaje cervical profiláctico. Indicaciones del cerclaje cervical profiláctico en los principales artículos analizados

\begin{tabular}{|l|l|l|}
\hline \multicolumn{1}{|c|}{ Artículo } & \multicolumn{1}{c|}{ Autores } & \multicolumn{1}{c|}{ Indicación de cerclaje } \\
\hline $\begin{array}{l}\text { Final report of the Medical Research } \\
\text { Council/Royal College of Obstetricians } \\
\text { and } \\
\begin{array}{l}\text { GynaecologistsMulticentreRandomised } \\
\text { Trial of Cervical Cerclage }\end{array}\end{array}$ & $\begin{array}{l}\text { M. C. Macnaughton, } \\
\text { I.G Chalmers, V. }\end{array}$ & $\begin{array}{l}\text { Debe ofrecerse a mujeres con alto riesgo } \\
\text { como antecedente de tres o más embarazos } \\
\text { finalizados antes de las 37 semanas }\end{array}$ \\
\hline $\begin{array}{l}\text { Cervical stitch (cerclage) for preventing } \\
\text { preterm birth in singleton pregnancy } \\
\text { (Review) }\end{array}$ & $\begin{array}{l}\text { Alfirevic Z, Stampalija } \\
\text { T, Medley N }\end{array}$ & $\begin{array}{l}\text { No hubo evidencia de ningún efecto } \\
\text { diferencial del cerclaje basado en } \\
\text { antecedentes obstétricos o indicaciones de } \\
\text { cuello uterino corto }\end{array}$ \\
\hline $\begin{array}{l}\text { Prevención del parto prematuro por } \\
\text { cerclaje del cuello uterino }\end{array}$ & L.Marcellin & $\begin{array}{l}\text { Se indica para embarazos únicos con } \\
\text { antecedentes de al menos tres abortos } \\
\text { espontáneos tardíos o partos prematuros, } \\
\text { no en otros antecedentes }\end{array}$ \\
\hline $\begin{array}{l}\text { Multicenter randomized trial of cerclage } \\
\text { for preterm birth prevention in high-risk } \\
\text { women with shortened midtrimester } \\
\text { cervical length }\end{array}$ & John Owen, Gary \\
\hline $\begin{array}{l}\text { The role of cervical cerclage in obstetric } \\
\text { practice: Can the patient who could benefit } \\
\text { from this procedure be identified? }\end{array}$ & $\begin{array}{l}\text { Roberto Romero, Jimmy } \\
\text { Espinoza, Offer Erez }\end{array}$ & $\begin{array}{l}\text { En mujeres con parto prematuro } \\
\text { espontáneo previo, menos de 34 semanas y } \\
\text { longitud cervical menor a 25 mm } \\
\text { cervical y disminución en longitud cervical } \\
\text { en ultrasonido se beneficia de cerclaje } \\
\text { cervical }\end{array}$ \\
\hline
\end{tabular}




\section{DISCUSIÓN}

Una de las causas más importantes del parto pretérmino de abortos tardíos es la incompetencia cervical, definida como la incapacidad del cuello uterino para retener un embarazo en ausencia de contracciones o trabajo de parto (4). Dado que la presentación de la misma suele darse de forma silente y repetida, se ha considerado la posibilidad de identificar población en riesgo y tomar medidas preventivas para evitar estos desenlaces desfavorables.

Han sido motivo de discusión las indicaciones para realizar un cerclaje preventivo que genere un beneficio, más que un riesgo de intervención quirúrgica no justificada. Inicialmente se consideró la historia obstétrica como suficiente indicación quirúrgica, planteamiento soportado por autores como Macnaughtony y colaboradores quienes resaltan el requerimiento de cerclaje profiláctico en toda mujer con antecedente de tres o más embarazos con finalización prematura $(12,21,24)$. Sin embargo, algunos autores consideran que el tipo de prevención debe depender del tipo de antecedente obstétrico $(17,18)$, siendo imperativa la presencia de al menos uno para realizar un método de prevención $(19,20)$. Aun así, autores como Abenhaimy y colaboradores resaltan que la historia obstétrica no es un parámetro suficiente para definir el requerimiento de cerclaje como medida preventiva (1).

Según el metanálisis realizado por Berglella y colaboradores, la longitud cervical fue el parámetro más importante identificado, siendo la historia obstétrica un parámetro que podría o no estar presente, siendo considerado como antecedente de importancia el aborto tardío previo, el parto prematuro y la conización $(2,3)$. To y colaboradores, reducen el valor de la cervicometría por ultrasonido como indicación preventiva al plantear que aun siendo esta menor a $15 \mathrm{~mm}$ si la historia obstétrica previa no es de alto riesgo, realizar un cerclaje para prevención no tendrá efectividad reduciendo el parto pretérmino espontáneo.
Considerando la probabilidad de ruptura prematura de membranas, amenaza de aborto, hemorragia anteparto e infecciones, entre otras posibles complicaciones derivadas de la realización del cerclaje cervical $(6,7)$, diferentes autores coinciden con la idea de no precipitarse a realizar el procedimiento pero sí realizar un seguimiento estrecho de la longitud cervical en pacientes con factores de riesgo o con sospecha de insuficiencia cervical $(9,10)$. Se ha planteado la posibilidad de realizar cerclaje profiláctico en caso de presentar disminución progresiva del diámetro longitudinal cervical por ecografía $(11,16,22)$. Otros parámetros asociados a la incompetencia cervical han sido considerados para apoyar el requerimiento preventivo quirúrgico como, el estado inflamatorio del endocérvix con la medición alta de interleuquina8 en moco cervical ya que favorece la incidencia de parto pretérmino (8). Se consideró en la mayoría de artículos, la realización de cerclaje por vía vaginal por medio de las técnicas de McDonald y Hervet, por estar estandarizadas, sin embargo en los estudios no fueron comparadas las diferentes técnicas en relación con mayor o menor éxito de la realización de cerclaje.

\section{CONCLUSIONES}

El parto pretérmino y el aborto tardío son desenlaces de la gestación en donde tiene un papel importante la insuficiencia cervical; ya que se trata de un cuadro silente y repetido, es importante realizar seguimiento y detección de las gestantes candidatas para la implementación de medidas preventivas para evitar aumento en la morbimortalidad tanto materna como fetal $(3,12)$. Es importante resaltar que un mayor tiempo fetal in útero permite un mayor desarrollo multisistémico, lo que será representado como un recién nacido con mayor adaptabilidad al medio, con APGAR alto, menor probabilidad de requerimiento de asistencia ventilatoria y cardiaca o de requerimiento de intervenciones quirúrgicas. Se deben considerar inicialmente los antecedentes obstétricos que son sugestivos de incompetencia cervical, teniendo en cuenta que algunos de ellos se catalogan como de alto riesgo, por lo cual se debe ser más exhaustivo en las medidas de vigilancia y 
anticipación a un parto pretérmino, así mismo, ser menos permisivos y considerar el contexto de la paciente y el beneficio de realización de cerclaje cervical temprano $(2,6)$. Se considera que en toda paciente con cualquier antecedente obstétrico de riesgo moderado o bajo, se debe valorar la longitud cervical por ecografía cada semana hasta llegar a término y en caso de presentar una longitud menor a $25 \mathrm{~mm}$ o descenso progresivo de la longitud, realizar cerclaje cervical profiláctico $(21,24)$. En las pacientes que no presentan cambios cervicales, se pueden considerar medidas farmacológicas como medidas de prevención iniciales mientras no se presenten cambios cervicales (1). Los resultados encontrados pueden ser extrapolados a nuestra población y favorecer una conducta óptima teniendo presente que la detección temprana de la población en riesgo es un pilar fundamental, por lo que una vez se realiza la identificación, se debe individualizar cada paciente, evaluando así, el requerimiento de intervención que tenga en el momento. Se deben exponer los riesgos y beneficios del procedimiento ya que la decisión de la paciente es un factor importante a considerar.

CONTRIBUCIONES DE LOS AUTORES: RGR, ATAA: concepción y diseño del estudio, recolección y análisis de resultados, redacción del artículo, revisión crítica y aprobación de versión final, responsables de la veracidad e integridad del artículo.

CONFLICTOS DE INTERESES: ninguno que declarar

\section{REFERENCIAS}

1. Abenhaim H, Tulandi T. Cervical insufficiency: Re-evaluating the prophylactic cervical cerclage. The Journal of Maternal-Fetal \& Neonatal Medicine. 2009; 22(6): 510-516.

2. Owen J, Hankins G, Iams J, Berghella V, Sheffield $\mathrm{J}$, Perez-Delboy A et al. Multicenter randomized trial of cerclage for preterm birth prevention in high-risk women with shortened midtrimester cervical length. American Journal of Obstetrics and Gynecology. 2009;201(4): 375.e1-375.e8.
3. Akladios C, Sananes N, Gaudineau A, Boudier E, Langer B. Le cerclage cervical. Journal de Gynécologie Obstétrique et Biologie de la Reproduction. 2015; 44(8): 771-775.

4. Romero R, Espinoza J, Erez O, Hassan S. The role of cervical cerclage in obstetric practice: Can the patient who could benefit from this procedure be identified?.American Journal of Obstetrics and Gynecology. 2006; 194(1): 1-9.

5. To M, Alfirevic Z, Heath V, Cicero S, Cacho A, Williamson $\mathrm{P}$ et al. Cervical cerclage for prevention of preterm delivery in woman with short cervix:randomised controlled trial. The Lancet. 2004; 363(9424): 18491853.

6. Althuisius S, Dekker G, van Geijn H, Bekedam D, Hummel P. Cervical Incompetence Prevention Randomized Cerclage Trial (CIPRACT): Study design and preliminary results. American Journal of Obstetrics and Gynecology. 2000; 183(4): 823-829.

7. Sakai M, Shiozaki A, Tabata M, Sasaki Y, Yoneda $\mathrm{S}$, Arai T et al. Evaluation of effectiveness of prophylactic cerclage of a short cervix according to interleukin-8 in cervical mucus. American Journal of Obstetrics and Gynecology. 2006; 194(1): 14-19.

8. Guzman E, Forster J, Vintzileos A, Ananth C, Walters C, Gipson K. Pregnancy outcomes in women treated with elective versus ultrasound-indicated cervical cerclage. Ultrasound in Obstetrics and Gynecology. 1998; 12(5): 323-327.

9. To M, Palaniappan V, Skentou C, Gibb D, Nicolaides K. Elective cerclage vs. ultrasound-indicated cerclage in high-risk pregnancies. Ultrasound in Obstetrics and Gynecology. 2002; 19(5): 475-477.

10. Higgins S, Kornman L, Bell R, Brennecke S. Cervical surveillance as an alternative to elective cervical cerclage for pregnancy management of suspected cervical incompetence. The Australian and New Zealand Journal of Obstetrics and Gynaecology. 2004; 44(3): 228-232.

11. Macnaughton M, Chalmers I, Dubowitz V, Dunn $\mathrm{P}$, Grant A, McPherson $\mathrm{K}$ et al. Final report of the Medical Research Council/Royal College of Obstetricians and GynaecologistsMulticentreRandomised Trial of Cervical Cerclage. BJOG: An International Journal of Obstetrics and Gynaecology. 1993; 100(6): 516-523. 
12. Drakeley A. Cervical cerclage for prevention of preterm delivery: meta-analysis of randomized trials. Obstetrics \& Gynecology. 2003; 102(3): 621-627.

13. Drakeley A, Roberts D, Alfirevic Z. Cervical stitch (cerclage) for preventing pregnancy loss in women. Cochrane Database of Systematic Reviews. 2003.

14. Alfirevic Z, Stampalija T, Medley N. Cervical stitch (cerclage) for preventing preterm birth in singleton pregnancy. Cochrane Database of Systematic Reviews. 2017.

15. Berghella V, Saccone G. Cervical assessment by ultrasound for preventing preterm delivery. Cochrane Database of Systematic Reviews. 2019.

16. Vargas I. P, Vera P-G. C, Carvajal C. J. Cerclaje para la prevención de embarazo de pretérmino en mujeres con cérvix corto encontrado en ecografía transvaginal: un estudio randomizado. Revista chilena de obstetricia y ginecología. 2004;69(6).

17. Marcellin L. Prévention de l'accouchement prématuré par cerclage du col de l'utérus. Journal de Gynécologie Obstétrique et Biologie de la Reproduction. 2016;45(10):1299-1323.

18. Hedriana H, Lanouette J, Haesslein H, McLean L. Is there value for serial ultrasonographic assessment of cervical lengths after a cerclage?.American Journal of Obstetrics and Gynecology. 2008; 198(6): 705.e1-705.e6.

19. Rust O, Atlas R, Reed J, van Gaalen J, Balducci J. Revisiting the short cervix detected by transvaginal ultrasound in the second trimester: Why cerclage therapy may not help. American Journal of Obstetrics and Gynecology. 2001; 185(5): 1098-1105.

20. Scheib S, Visintine JF, Miroshnichenko G, Harvey C, Rychlak K, Berghella V. Is cerclage height associated with the incidence of preterm birth in women with an ultrasound-indicated cer- clage? Am J ObstetGynecol 2009; 200: e12-5.

21. Miller E, Gerber S. Association Between Sonographic Cervical Appearance and Preterm Delivery After a History-Indicated Cerclage. Journal of Ultrasound in Medicine. 2014; 33(12): 2181-2186.

22. Sim S, Da Silva Costa F, Araujo Júnior E, Sheehan P. Factors associated with spontaneous preterm birth risk assessed by transvaginal ultrasound following cervical cerclage. Australian and New Zealand Journal of Obstetrics and Gynaecology. 2015; 55(4): 344-349.

23. Berghella V, Keeler S, To M, Althuisius S, Rust O. Effectiveness of cerclage according to severity of cervical length shortening: a meta-analysis. Ultrasound in Obstetrics and Gynecology. 2010; 35(4): 468-473.

24. Owen J, Mancuso M. Cervical Cerclage for the Prevention of Preterm Birth. Obstetrics and Gynecology Clinics of North America. 2012; 39(1): 25-33.

25. Berghella V, Mackeen A. Cervical Length Screening with Ultrasound-Indicated Cerclage Compared with History-Indicated Cerclage for Prevention of Preterm Birth. Obstetrics \& Gynecology. 2011; 118(1): $148-155$.

26. Kagan K, To M, Tsoi E, Nicolaides K. Preterm birth: the value of sonographic measurement of cervical length. BJOG: An International Journal of Obstetrics \&Gynaecology. 2006; 113: 52-56.

27. Cook J, Chatfield S, Chandiramani M, Kindinger L, Cacciatore S, Sykes L et al. Cerclage position, cervical length and preterm delivery in women undergoing ultrasound indicated cervical cerclage: A retrospective cohort study. PLOS ONE. 2017; 12(6): e0178072. 